\title{
A Taxonomy for Advanced Public Transportation Systems
}

\author{
Asad J. Khattak \\ University of North Carolina at Chapel Hill \\ Hisham Noeimi \\ JRH Transportation Engineering \\ Haitham M. Al-Deek \\ University of Central Florida
}

\begin{abstract}
This study explores the development and availability of APTS (Advanced Public Transportation Systems) technologies. APTS technologies can revitalize transit by directly improving service, increasing transit efficiency and reducing operating costs, as well as by producing direct benefits for travelers such as reduced travel times, increased safety and security, and reduced stress in dealing with transit unreliability. To understand APTS impacts, this study develops a taxonomy of transit technologies and uses it to explore the availability of new technologies and their impacts. The taxonomy is based on defining the features, functions, and performance characteristics of transit technologies. Further, the implementation of new technologies can be described by their spatial, temporal, and user dimensions, i.e., where, when, and for whom is the technology implemented. These dimensions, along with the implementation context, determine the impacts of APTS technologies. To explore the availability of APTS technologies, technology suppliers were surveyed. They were asked about the features, functions, and perfor-
\end{abstract}


mance of transit technologies, their testing and deployment in transit agencies, and their potential impacts on travelers and transit operators. The survey results suggest a trend toward transfer of data in real-time through electronic media and increased automation. It was found that about a dozen APTS technologies queried in the survey were commercially available for field testing. From a policy perspective, there is a need to develop a strategy that considers the individual and joint testing of two or more APTS technologies and facilitates synthesis of the resulting information. Individually, the benefits of APTS technologies may be limited, but, collectively, APTS technologies may have significant benefits. Cases of joint APTS technology implementations need to be designed, implemented and synthesized.

\section{Introduction}

While traffic congestion grows, public transportation continues to lose market share in the United States. Specifically, the share of transit trips shows a declining trend: 3.6 percent in 1969, 2.6 percent in 1983 and 2 percent in 1990 (Pisarski 1992). Moreover, the use of public transportation for work travel has declined from 12.6 percent in 1960 to 5.3 percent in 1990 (Ball 1994). Recent advances in electronic technologies may allow greater integration of transit services and increase transit use. Advanced Public Transportation System (APTS) technologies may increase transit efficiency, improve transit level of service, reduce costs, and avoid further reductions in transit use. To assess the potential of APTS technologies, there is a need to systematically explore their impacts (Khattak et al. 1993). The main objectives of this study are to:

- define and classify APTS technologies and identify their impacts;

- use the classification structure for exploring availability of new transit technologies; and

- provide ideas on individual and joint testing of APTS technologies.

The structure developed to classify and investigate the availability of new transit technologies is based on defining technologies in terms of their features, functions, and performance. For example, one feature of transit information technology is the communication medium (whether information is disseminated through visual or audio means); a function is provided by the content of dissemi- 
nated information (subject matter and whether the information is historical or real-time); and a key performance measure is information quality (accuracy and relevance).

To support the implementation of APTS technologies, their spatial, temporal, and user dimensions should be defined. For example, the spatial dimensions of transit information technologies are the transit vehicles that are monitored and the links served. The temporal dimensions are the free-flow travel times on transit links and the times monitoring is in effect. The user dimensions are whether certain travelers access transit information devices and actually choose to take transit.

APTS technologies can be traveler-based, operator-based, or both. The traveler-based technologies influence traveler behavior directly but can indirectly impact operators (e.g., pre-trip or in-terminal information systems). Similarly, operator-based technologies influence transit operators directly and travelers indirectly (e.g., Automatic Vehicle Monitoring systems). Mixed technologies simultaneously impact both travelers and operators. Finally, the technologies are implemented, and the impacts occur in a context characterized by the spatial, temporal, and user dimensions. For example, the transportation network structure, its state at various times, and population characteristics (density and socioeconomics) can be important determinants of APTS impacts.

The following section describes the process of transit technology implementation. Then, taxonomies for new transit technologies and their impacts are discussed. Next, the development and implementation of a transit technology supplier survey and results are presented. Finally, conclusions are drawn and the need to develop a strategy for systematically testing new transit technologies is identified.

\section{Process of Technology Implementation}

Figure 1 shows the process of technology supply, demand, and implementation. The demand for transit technologies may come from the political process, which can encourage the use of APTS technologies. For example, the ISTEA (Intermodal Surface Transportation and Efficiency Act) legislation encourages 


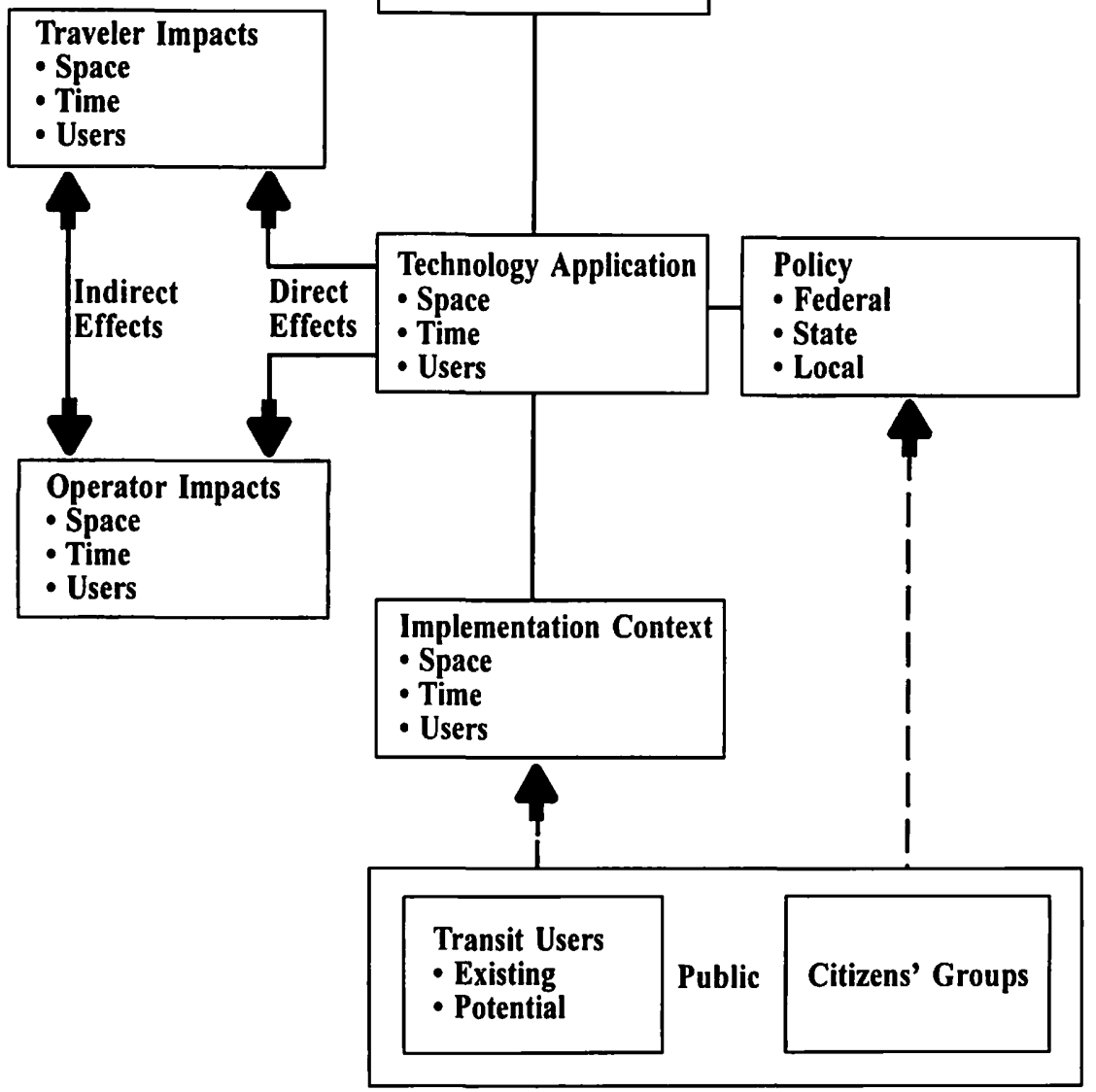

Technology

Developers/Suppliers

Technology

- Features

- Functions

- Performance

Implementation Context

- Space

- Time

- Users

- Existing

Citizens' Groups

Figure 1. The process of technology supply, demand, and implementation. 
multimodal systems because they can achieve certain goals such as reduce traffic congestion and pollution. Further, ISTEA encourages increased transit security by giving incentives. The Americans with Disabilities Act (ADA) promotes the use of certain technologies because it mandates accessibility for the disabled. Besides the federal policies, state and local policies create the demand for new transit solutions. The demand for improved technology in transit systems is also stimulated by the public, i.e., existing and potential transit users and citizens' groups (who advocate transit improvements).

Transit technology developers and suppliers respond to the market (or sometimes create a market) by designing new technologies using advances in electronics and machines. To satisfy demand, suppliers develop technologies that have certain features, functions, and performance characteristics. Table 1 gives a summary of advanced transit information technologies in terms of their features, functions and performance criteria (Khattak et al. [1993] provide similar summaries for other transit technologies). The technology features, functions and performance measures are based on a review of literature and our judgment (see Khattak et al. [1993] for a comprehensive review of relevant literature). Table 1 suggests that pre-trip information systems can disseminate information by several means, including telephone, computer, and television. Therefore, the medium of information dissemination is important in technology definition. Transit information systems provide historical and real-time information on transit operations (routes, schedules, and fares) to travelers and some systems do advance ticketing and reservation. Therefore, the content of information and other functions are important. Moreover, the accuracy and relevance of information provided is likely to vary. The quality of information is important in traveler decisionmaking. Thus, a set of design factors for pre-trip information technologies that partly determine impacts are the medium, content, and quality of information.

The spatial, temporal, and user dimensions of the technologies and the implementation context influence impacts. The technology application takes place in an implementation context defined by the transit agency characteristics such as service to certain populations (e.g., commuters, lower income, disabled) in a 


\section{Table 1}

\section{Characteristics of Selected Advanced Transit Information Technologies}

\begin{tabular}{|c|c|c|c|}
\hline Technology & $\begin{array}{c}\text { Features } \\
\text { (Information } \\
\text { Medium) }\end{array}$ & $\begin{array}{l}\text { Functions } \\
\text { (Information } \\
\text { (content) }\end{array}$ & $\begin{array}{c}\text { Performance } \\
\text { (Information } \\
\text { Quality) }\end{array}$ \\
\hline $\begin{array}{l}\text { Pre-Trip } \\
\text { Information } \\
\text { Systems }\end{array}$ & $\begin{array}{l}\text { - Auto dial phone } \\
\text { - Telephone to } \\
\text { computer operator } \\
\text { - Voice recognition } \\
\text { - Computer \& modem } \\
\text { - Teletext } \\
\text { - Videotext } \\
\text { - Audiotext } \\
\text { - Cable TV } \\
\text { - Interactive voice } \\
\text { response } \\
\text { - Interactive TV }\end{array}$ & $\begin{array}{l}\text { - Provides historical } \\
\text { or real time information: } \\
\text { - schedule/departure times } \\
\text { - multimodal itinerary } \\
\text { - trip chaining (itinerary } \\
\text { optimization) } \\
\text { - ride share opportunities } \\
\text { - best route based on } \\
\text { traveler criteria: } \\
\text { - shortest time } \\
\text { - lowest fare } \\
\text { - intermediate stops } \\
\text { - maximum use of } \\
\text { rapid transit } \\
\text { - least walking distance } \\
\text { - Provides advance } \\
\text { ticketing \& reservations }\end{array}$ & $\begin{array}{l}\text { - Presentation quality } \\
\text { - Accuracy of information } \\
\text { - Relevance of information }\end{array}$ \\
\hline $\begin{array}{l}\text { In-Terminal } \\
\text { Information } \\
\text { Systems }\end{array}$ & $\begin{array}{l}\text { - Dot matrix display } \\
\text { - Flipover display } \\
\text { - LCD } \\
\text { - TV monitors } \\
\text { - Synthesized } \\
\text { voice messages } \\
\text { - Audio terminals } \\
\text { - Video terminals: } \\
\text { - with keypads } \\
\text { - with touch screens }\end{array}$ & $\begin{array}{l}\text { - Provides historical or } \\
\text { real-time information: } \\
\text { - schedule/departure times } \\
\text { - multimodal itinerary } \\
\text { - trip chaining (itinerary } \\
\text { optimization) } \\
\text { - ride share opportunities } \\
\text { - best route based on } \\
\text { traveler criteria: } \\
\text { - shortest time } \\
\text { - lowest fare } \\
\text { - intermediate stops } \\
\text { - maximum use of } \\
\text { rapid transit } \\
\text { - least walking distance } \\
\text { - connection points } \\
\text { - transit vehicle location/ } \\
\text { delays information } \\
\text { - terminal related informat } \\
\text { (e.g., layout) } \\
\text { - destination } \\
\text { - Provides advance ticketing } \\
\text { \& reservations }\end{array}$ & $\begin{array}{l}\text { - Presentation quality } \\
\text { - Accuracy of information } \\
\text { - Relevance of information }\end{array}$ \\
\hline
\end{tabular}

Source: Khattak et al., 1993 
specific area, on certain routes, and at specific times (e.g., frequencies and schedules). In addition to the implementation context, the technology is also defined in terms of space, time, and user dimensions. For example, Automatic Vehicle Monitoring (AVM) systems report transit vehicle location on specific network links at certain times, and the position information of vehicles is relevant to supervisors who make operations decisions (e.g., advise drivers on maintaining headways and schedules). To refer to various aspects of APTS technologies, the term "technology space" is defined as having design dimensions (features, functions, and performance) and application dimensions (space, time, and user). Equivalently, "impacts space" is defined in terms of performance criteria or dimensions (efficiency, service quality, cost, time savings) and distribution dimensions (space, time and users). Technologies can have direct, indirect, and simultaneous impacts on operators and travelers. For example, transit operations software, AVI (Automatic Vehicle Identification) and AVL (Automatic Vehicle Location) systems are expected to have strong direct impacts on transit operators. Transit information systems are expected to directly influence travelers. The following direct transit operator impacts occur:

- reduced costs such as maintenance, fuel, labor, management and marketing costs, and

- improved efficiency through better transit planning and operations-

- the planning functions that can be improved include the selection of service area, routes, stops, and service frequencies, and

- the operations improvements can come from better ability to monitor driver and vehicle performance, improved scheduling and dispatching, reduced human errors, improved fare structure, and enhanced safety and security.

The indirect benefits of transit improvements accrue to transit travelers (and non-transit travelers through reduced congestion and pollution on highways). Impacts from APTS technologies are distributed in space and time and by various types of operator decisions. The magnitude of direct operator impacts depends on the technology design dimensions, technology application dimensions, and the implementation context. 
The following direct traveler benefits due to APTS technologies can be expected:

- travel time savings and reduced uncertainty in travel times;

- improved accessibility;

- improved content, medium and quality of transit information;

- increased flexibility in travel choices;

- improved (accident) safety and security;

- ease of transit use, improved travel comfort and convenience; and

- improved satisfaction with transit service and customer feedback.

The key indirect benefit to a transit agency is increased ridership. The impacts from individual APTS technologies can vary across the impacts space, i.e., the traveler impacts are distributed in space, time, and by different travelers. Sometimes, APTS technologies may influence travelers differently by design. For example, a technology that enhances ease of transit use may be particularly appealing to the elderly and disabled, whereas a technology that increases travel choices may be attractive for shoppers (because of opportunities to shop at more destinations). The extent of direct traveler impacts depends on the technology design and application dimensions and the implementation context.

This study explores the availability of newly developed transit technologies for field testing in transit agencies. There are several projects in the United States aiming to test different advanced technologies (see Khattak et al. 1993). For example, the Federal Transit Administration (FTA) and California Department of Transportation (Caltrans) are co-funding a four-phase California Smart Traveler Project where public and private sectors will jointly test an audiotext/videotextbased Advanced Traveler Information System in suburban California. We hope to help the development of such projects through research on the features, functions, and performance of new transit technologies and through suggestions on deployment strategies. This requires that the technologies and their implementation context and impacts be defined in terms of spatial, temporal, and user dimensions. Importantly, knowledge and models are needed to determine the impacts of APTS technologies individually and collectively. 


\section{Technology Definition and Evaluation}

\section{Traveler Information Systems}

Information content, medium, and quality can influence various traveler choices. The taxonomy with regards to information content is explained below. Information can be either static or dynamic. Static information related to travel choices does not change with time, whereas dynamic information changes with time. Information can be further divided into qualitative or quantitative. Infor-

Technology:

Manufacturer/Sponsor:

\begin{tabular}{|l|l|l|l|l|}
\hline \multirow{2}{*}{$\begin{array}{l}\text { Information } \\
\text { Traveler } \\
\text { Choices }\end{array}$} & \multicolumn{2}{|c|}{ Static } & \multicolumn{2}{c|}{ Dynamic } \\
\cline { 2 - 5 } & Qualitative & Quantitative & Qualitative & Quantitative \\
\hline Destination & & & & \\
\hline Multimodal & A & B & C & D \\
\hline Departure Time & & & & \\
\hline Route & & & & \\
\hline Park and Ride & & & & \\
\hline Trip Chaining & & & & \\
\hline
\end{tabular}

Technology Functions:

$\begin{array}{lll}\text { Multimodal Reservation } & \square \text { Yes } & \square \text { No } \\ \text { Integrated Billing System } & \square \text { Yes } & \square \text { No } \\ \text { Seating Availability } & \square \text { Yes } & \square \text { No }\end{array}$

Information Medium:
$\square$ Portable
$\square$ Non-Portable
$\square$ In-Vehicle
๑ Out-of-Vehicle
$\checkmark$ Audio
$\square$ Visual

Figure 2. Taxonomy of traveler-based transit information systems. 
mation content and travel choices form a two dimensional taxonomy matrix (Figure 2). As an illustration of this matrix, consider the following examples:

- Static Qualitative, Multimodal Information (Cell " $A$ "). Static information about the availability of trip connections may support multimodal choice. For example, based on trip connections information, a traveler may use bus instead of auto to reach the nearest train station.

- Static Quantitative, Multimodal Information (Cell " $B$ "). Static information about transit schedules can reduce wait times and support mode choice.

- Dynamic Qualitative, Multimodal Information (Cell " $C$ "). Real-time information about whether a bus is on-time can support the choice of using a bus or walking to a train station.

- Dynamic Quantitative, Multimodal Information (Cell " $D$ "). Real-time information about expected arrival times of the next bus or train and expected delays can support travelers' modal choice.

In addition to static and dynamic information, transit systems may provide predictive information such as the expected time to recovery of a breakdown. Other functions provided by information systems are integrated billing service and multimodal (park-and-ride) trip reservation.

Information medium is important in determining traveler impacts. Whether a device is portable or fixed (and if fixed, whether it is in-vehicle or out-ofvehicle) and visual or audio are important aspects of traveler information technologies. Furthermore, information quality is an important performance criterion. Clearly, individuals prefer higher quality information.

From a technology implementation perspective, the spatial, temporal and user dimensions are important. Specifically, where and when the information technology is implemented and who are the expected users is important in determining its impacts. The spatial dimensions of transit information technologies are the transit vehicles that are monitored through surveillance technologies and the relevant routes. The temporal dimensions are the free-flow travel times on transit links and the times vehicles are monitored. The user dimensions are whether travelers access transit information devices and decide to take public transit. 


\section{Rideshare Systems Technologies}

Real-time rideshare matching systems allow trip makers to call in for sharing a ride either as drivers or as passengers. Rideshare matching software allows travelers to review rideshare options, identify individuals whose needs closely match their own, and reserve the trip in advance. Real-time rideshare systems will provide information on other travelers to potential ridesharers. The taxonomy matrix can be used to understand how travel decisions may be influenced by the content of rideshare information. The following information can be provided by a rideshare information service (for high occupancy vehicles):

- Static-Qualitative Information. Examples of static-qualitative information are potential candidates for rideshare, location of candidates' homes and their preferences.

- Static-Quantitative Information. Examples of static-quantitative information are preferred times of departure and distance to homes of the candidates.

- Dynamic-Qualitative Information. The rideshare system may inform customers of delays due to personal emergencies.

- Dynamic-Quantitative Information. The service may give information on the number of persons available at certain times of the day, expected length of delays, and dynamic travel time information for HOV (High Occupancy Vehicle) and mixed-flow lanes.

\section{Automatic Vehicle Control Technologies}

Early versions of Automatic Vehicle Control System technologies provide driver warning and assistance, resulting in collision avoidance. The technologies can perform collision avoidance by obstacle detection, lane edge warning, and some level of lateral/longitudinal control. These systems are in their early stages of development. They use radar, infrared laser, or sonar and provide either warning only or warning with braking. They can improve transportation safety by reducing accidents. The information provided to drivers is dynamic. It can be qualitative, such as "You are very close to the right edge of the lane," or quantitative, such as "You are $x$ feet away from the vehicle in the right lane." The 
information will be disseminated through visual, audio, or both means. The content, medium, and quality of information will influence the driver response to the warnings.

\section{Automatic Vehicle Monitoring, Automatic Ticketing,}

\section{Automatic Passenger Counters, and On-Board Computer Systems}

To support supervision and coordination, certain technologies provide surveillance and monitoring. Automatic Vehicle Monitoring systems can simultaneously improve transit operations (dispatching, scheduling, and security) while providing real-time transit system operation information to travelers. Electronic Ticketing Systems (ETS) automate fare collection, increasing convenience and adding modest travel time savings to a trip. Automatic Passenger Counters (APC) send passenger counts to a central facility in real-time. Together, APC and ETS provide valuable data to transit operators on passenger loads and schedule adherence. These data can be used to support transit operations (dispatching and scheduling) and planning.

A taxonomy of technologies can be applied to the information that comes from various technology sources to a central transit management center. The operator decisions that can be supported include operations (dispatching, scheduling, supervision, monitoring, coordination, and fare collection) and planning (area and routes to serve, service type-regular or express, stops, frequencies, fare structure, and maintenance). The content of information and its medium and quality are likely to influence operator decisions. In addition, the analysis techniques used to process information (e.g., expert systems and breakdown duration prediction models) influence operator decisions. The information can be historical (qualitative or quantitative) or real-time (qualitative or quantitative). For example, real-time information about the location of buses and whether they are on time is available to operators through AVI and AVL systems. Such information can be used to avoid bunching, detect breakdowns, and disseminate the information to travelers. On-board computers collect vehicle data (oil, water, engine temperature, vehicle speed, etc.), which can be used by the driver and transit operators to monitor vehicle performance and detect and deal effectively with breakdowns. Transit operations software supports transit planning decisions of ve- 
hicle and crew scheduling, maintenance, and marketing. The software can also be connected to AVI/AVL systems for greater effectiveness.

More generally, the information and how transit operators choose to process it can support their decisions. The information can relate to transit system performance, traffic system performance, and traveler demand at various origins and destinations and times of day.

\section{Survey Methodology}

The objectives of this study are to define APTS technologies, track their development, and suggest a strategy to evaluate their impacts on transit operators and travelers. Ideally, the study should be designed to address these objectives simultaneously. However, when the study commenced, APTS technologies were still under development and not implemented by transit agencies. Therefore, a decision was made to survey technology suppliers during the first phase of the study. The subsequent phases focus on surveying technology implementers and travelers (and these phases are ongoing). The remainder of this section presents the methodology, and the next section reports the results of the technology supplier survey.

The methodology for the technology supplier survey is illustrated in Figure 3. After classifying Advanced Public Transportation Systems (APTS) according to their features, functions, and performance, a survey was designed to explore their (commercial) availability. The survey also inquired (from technology suppliers) about supplier attributes, the application of their technologies in transit agencies, and their perceived impacts on travelers and transit operators. The survey was structured as follows:

- available APTS technologies defined in terms of features, functions and technology performance;

- technology deployment in transit agencies, i.e., "typical customers" and customer attributes;

- perceived impacts of technologies on operators and travelers; and

- technology supplier attributes. 


\section{Objective}

- Develop a framework for classifying APTS technologies

- Explore their impacts and availability

\section{$\checkmark$}

Address Objective

- Develop structure based on technology features, functions, and performance

- Implementation of technologies based on temporal, spatial, and user dimensions

- Use survey research to investigate availability of advanced transit technologies

- APTS technology

\section{Design Survey Based On}

- Features

- Functions

- Performance

- Technology implementation in transit agencies

- Typical customers

- Customer attributes

- Technology Impacts

- Operator benefits

- User benefits

- Technology supplier/developer attributes

\section{Pre-Test and Implement Survey, Code and Analyze Data}

Figure 3. Study methodology. 
About 100 surveys were mailed to technology suppliers; 20 were used for analysis $(\mathrm{N}=20)$. The respondents included most major APTS companies in the United States. The details of the survey appear in Khattak et al. (1993). The purpose of these surveys was not so much to conduct formal statistical comparisons, but to obtain information on available transit technologies.

\section{Results}

Responses were spread evenly across technology categories. Given the comprehensive list of APTS technologies explored in the survey, this suggests that most APTS technologies are commercially available for field testing. As a result of the survey, a database of technology suppliers in the United States was created.

The responses suggest that both small and large companies are competing in the APTS technology market. Most respondents manufactured their products in the U.S., and most fabricated their products in-house. Supplier responses to each transit technology are presented below.

\section{Pre-Trip Information Systems}

Ten companies sell pre-trip information systems: Schlumberger Technologies, Tidewater Inc., Etak Inc., Qualcomm Inc., Megadyne Info Systems, Fone Link Inc., Commuter Transportation Services, Teleride Sage Ltd., Westinghouse, and Peek Traffic. For the information medium, 5 companies use automatic dial phone technology, 6 use telephone to computer operator technology, 2 use telephone and voice recognition technology, 5 use computer modem technology, 7 use teletext, 4 use videotext, 1 uses cable TV, and 4 systems use other kinds of technology. In terms of the information content provided to the traveler, 9 have systems that provide schedule/departure times ( 3 are based on historical information and 6 are real time); 7 provide multimodal itinerary information; 3 provide trip chaining (itinerary optimization) information ( 3 are historical and 4 are real time); and 6 provide information on rideshare opportunities ( 4 are historical and 2 are real time). Further, the systems can provide best route information based on traveler selected criteria, which include: (i) shortest time6 of the suppliers, (ii) lowest fare- 6 , (iii) intermediate stops- -5 , (iv) maximum 
use of rapid transit-5, and (v) least walking distance- 6 . In addition, 8 of the systems provide information on connection points, and 7 provide information about transit vehicle location. In terms of a map base, only 1 uses Etak, 5 use Tiger, and 4 use other map bases. Only 1 system provides advance ticketing and reservation capability.

\section{In-Terminal Traveler Information Systems}

Seven suppliers have in-terminal information systems. The suppliers include: Tidewater Inc., Westinghouse, Etak Inc., Megadyne Information Systems, Teleride Sage Ltd., Peek Traffic, and Midwest Electronic Industries. Of the available systems, six disseminate information by dot matrix displays (6), 4 use flipover displays, 4 use Liquid Crystal Displays (LCD), 2 use TV monitors, 5 use synthesized voice messages, 2 use interactive audio terminals, 3 use interactive video terminals with keypads, and 4 use interactive video terminals with touch sensitive screens.

In terms of information content, 4 provide schedule/departure times ( 2 are historical and 2 are real time), 5 provide multimodal itinerary information, 3 provide trip chaining information (itinerary optimization), and 3 provide rideshare opportunities information. The systems can provide best route information based on traveler selected criteria, which include: (i) shortest time- 3 (2 are historical and 1 is real time), (ii) lowest fare-3, (iii) intermediate stops-2, (iv) maximum use of rapid transit -3 , and ( $v$ ) least walking distance-3. All available systems provide information on connection points, 6 provide information on transit vehicle location, 5 provide information on delays, and all provide information on the destination. Only 1 available system uses Etak as the map base, 4 use Tiger, and 2 use other map bases. None provide advanced ticketing and reservation, and all available systems can be linked to other sources of information.

\section{In-Vehicle Traveler Information Systems}

Six out of 20 companies have in-vehicle information systems: AEG Westinghouse, Motorola, Etak Inc., Megadyne Info Systems, Peek Traffic, and Midwest Electronic Industries. Among the available systems, 5 use synthesized voice messages to disseminate information, 5 use dot matrix displays, 3 use video 
displays, 2 use flap displays, and 2 use other technologies. In terms of information content provided to travelers, 5 give schedule information ( 2 are historical and 3 are real time), 4 provide the expected arrival time at the next stop ( 1 is historical and 3 are real time), 3 provide waiting times at connecting points, 4 provide connecting services, 1 provides seating availability information, and 4 provide next stop announcements. Only 1 available system uses Etak as the map base, 7 use Tiger, and 10 use other map bases. None provide advance ticketing and reservation.

\section{Rideshare Matching Software}

Five suppliers provide real-time rideshare matching software: Tidewater Inc., Comsis, Megadyne Information Systems, Fone Link Inc., and Commuter Transportation Services. A majority (3) provide real-time matching, and 4 use Tiger as their map base. Two of the latest systems match passengers by grid, and 2 match them by zip code.

\section{Automatic Vehicle Identification}

Six suppliers have AVI technologies: Amtech Technologies, EMX Inc., AEG Westinghouse, Fone Link Inc., LazerData, and Peek Traffic. Among the available technologies, 2 are based on Infrared/Optical, 3 on Radio Frequency (RF)/ Microwave, 2 on Inductive Loop, and 1 on Surface Acoustic Wave (SAW). Four out of 6 have two-way communications capability between reader unit and vehicle mounted transponder, and 1 encodes variable data. In terms of technology performance, 1 respondent claimed that its system misses no vehicles, and the rest miss less than 1 percent of the vehicles.

\section{Automatic Vehicle Location}

Ten of the suppliers sell AVL systems: EMX Inc., AEG Westinghouse, Motorola, Etak Inc., Qualcomm Inc., Megadyne Information Systems, Rockwell RR Electronics, II Morrow Inc., Teleride Sage Ltd., and Peek Traffic. Of the systems on the market, only 1 uses dead reckoning, 2 use dead reckoning with map matching, 4 use GPS with dead reckoning, 2 use GPS with map matching, 2 use Proximity Beacon Sign Post, (all of which use "sharp" transmissions [localized signals] as opposed to "broad" transmissions [long range signals]), 4 use 
Radio Determination ( 2 of which use Loran-C), 5 use Satellite Based systems (4 of which are GPS/NAVSTAR), and 5 systems use other methods.

In terms of technology performance, 2 track the location of the transit vehicle within 30 feet, 60 percent track it between 30 and 100 feet, 1 tracks it between 100 and 500 feet, and 1 tracks it at more than 500 feet. Four of 8 systems update location information every 1 second, 1 updates information every 30 seconds, 2 update it every 60 seconds, and 1 updates the position information every 60 minutes.

\section{Collision Avoidance Systems}

Only two companies have collision avoidance systems: Westinghouse and Rockwell RR Electronics. Both provide warning and braking functions.

\section{On-Board Computers}

Seven vendors have on-board computers: Westinghouse, Etak Inc., Qualcomm Inc., Pulse Electronics, Rockwell RR Electronics, II Morrow Inc., and Peek Traffic. The computers collect data on speedometers (6), and ignition status (on/off) (5). Most systems (6) are connected to a central computer for data integration and processing. Only 1 system each uses Etak and Tiger as the map base, and the remaining 5 use other map bases.

\section{Transit Operations Software}

Three suppliers provide transit operations software. They include: Westinghouse, Megadyne Information Systems, and Teleride Sage Ltd. None of the available systems provide marketing functions; only 1 provides management and administration functions; 1 provides network and operations planning based on historical and real-time information; and 2 provide vehicle and crew scheduling. Two use Tiger as their map base. Two of the latest transit operations software systems can be connected to an Automatic Vehicle Location system, and 2 can be connected to Automatic Vehicle Identification systems.

\section{Electronic Ticketing Systems}

The companies that supply Electric Ticketing Systems include Schlumberger and AEG Westinghouse. Both systems collect origin destination data and revenue information disaggregated by route and ticket type; 1 collects passenger 
information disaggregated by class, route, and time of day. In terms of payment, 1 system can accept credit cards; both have tickets that can be reused by adding fare to them; 1 has tickets good for one ride only. Both have tickets good for a limited number of rides, whereas 1 has tickets good for unlimited rides. Moreover, 1 of the ETS has tickets that the traveler can use for multimodal transportation.

\section{Automatic Passenger Counter}

The suppliers who have Automatic Passenger Counters are Westinghouse, Red Pine Instruments, and Peek Traffic. One company provides a pressure-sensitive mat for counting, and the rest use infrared beams. All use random access memory (RAM) to store information. A majority (2) send passenger counts at bus stops to the dispatcher in real time. One company reported that their system misses less than 1 percent of passengers and provides information on the total number of passengers served along a route, the actual number of passengers on the bus, and the number of passengers boarding and alighting at certain stops.

\section{Automatic Demand-Responsive Dispatching Systems}

Six suppliers have automatic demand responsive dispatching systems. All provide scheduling functions ( 4 are based on historical data and 2 on real time data); all provide dispatching ( 4 are based on historical data and 2 on real time data); 3 provide billing functions; and 5 provide service monitoring and reporting. Four consider traveler preferences, 5 provide transit vehicle location information to the traveler in real-time, 4 provide best route information according to traveler selected criteria, 2 have reservations capabilities, 5 can respond to immediate requests, and all can respond to standing orders. Two of the systems use Tiger as their map base, and 3 use other map bases.

\section{Impacts of APTS Technologies}

Casey and Collura (1993) recognize the need to have a consistent and carefully structured approach to operational test evaluation. In this study, the factors that will influence the outcomes of APTS field tests are hypothesized, and the potential impacts are examined from the technology suppliers' perspective. The true impacts of new technologies will depend on the design and application di- 
mensions of the APTS technologies and the implementation context. Further, the traveler and operator impacts will vary across space, time, and users. Ultimately, knowledge and models are needed to relate APTS technologies and the implementation context to specific traveler and operator impacts. Such knowledge can come from designing and examining case studies. Technology implementation decisions can be made based on historical evidence from field tests; successful field tests can be replicated and unsuccessful ones avoided (Khattak and Kanafani 1995). The implementation decisions can also be made by specifying the inputs (technologies and their implementation context) and the desired outputs (operator and traveler impacts) and running models. The models can evaluate impacts for alternative technology design and application scenarios. However, APTS knowledge is scarce, and models do not exist for APTS evaluation. This study, by collecting information about available APTS technologies, is one effort in the direction of building an APTS knowledge base. In this regard, the opinions of technology suppliers with respect to traveler and operator impacts are examined.

A simplified representation of technology and impact combination is shown in Table 2. It is simplified because no consideration is given to the implementation context and the distribution dimensions of impacts. The table represents scores given by vendors to their highest revenue technologies. The subjective technology evaluations (opinions) provided by the respondents are likely to be biased because the suppliers are selling these products. Also, the small sample size limits generalization. Yet the responses provide insights into the "relative" impacts across the categories and the potential for direct impacts. As expected, operator-based technologies get higher ratings on operator performance (e.g., monitoring, headways, labor hours, operating time, and human error), while traveler-based technologies get higher ratings on operator performance (e.g., user complaints, safety, travel flexibility).

The overall trend in new technologies is toward transfer of data in real-time through electronic media. Most APTS technologies can support either traveler decisions or transit operator decisions and, in some cases, both types of decisions.

In the field of advanced transit technologies, some relatively large companies, such as Schlumberger, Westinghouse, Motorola, and Rockwell, have en- 
Table 2 Ophions of APTS Technology Suppliers Regarding Their Main Transit Product

\begin{tabular}{|c|c|c|c|c|c|c|c|c|c|c|c|c|}
\hline $\begin{array}{l}\text { Tech- } \\
\text { nology }\end{array}$ & $\begin{array}{l}\text { Improved } \\
\text { Monitor- } \\
\text { ing }\end{array}$ & $\begin{array}{l}\text { Controls } \\
\text { Transit } \\
\text { Head- } \\
\text { ways }\end{array}$ & $\begin{array}{l}\text { Reduced } \\
\text { Labor } \\
\text { Hburs }\end{array}$ & $\begin{array}{c}\text { Reduced } \\
\text { Operating } \\
\text { Time }\end{array}$ & $\begin{array}{c}\text { Reduced } \\
\text { Human } \\
\text { Error }\end{array}$ & $\begin{array}{l}\text { Improved } \\
\text { Security }\end{array}$ & $\begin{array}{l}\text { Reduced } \\
\text { User } \\
\text { Com. } \\
\text { plaints }\end{array}$ & $\begin{array}{l}\text { Improved } \\
\text { Safety }\end{array}$ & $\begin{array}{l}\text { Increased } \\
\text { Travel } \\
\text { Flex- } \\
\text { ibility }\end{array}$ & $\begin{array}{c}\text { Easier to } \\
\text { Use } \\
\text { Transit }\end{array}$ & $\begin{array}{l}\text { Improved } \\
\text { Travel } \\
\text { Comfort }\end{array}$ & $\begin{array}{l}\text { Improved } \\
\text { User } \\
\text { Satis- } \\
\text { faction }\end{array}$ \\
\hline $\mathrm{AVI}$ & 3.00 & 3.00 & 3.33 & 3.00 & 3.66 & 3.00 & 2.33 & 3.33 & 3.00 & 3.33 & 3.66 & 3.66 \\
\hline AVL & 3.66 & 3.66 & 3.00 & 2.66 & 3.00 & 3.00 & 3.00 & 2.33 & 2.66 & 2.66 & 2.66 & 3.33 \\
\hline $\mathrm{OBC}$ & 4.00 & 2.00 & 2.00 & 2.00 & 4.00 & 3.00 & 2.00 & 4.00 & 2.00 & 2.00 & 2.00 & 2.00 \\
\hline $\mathrm{APC}$ & 2.00 & 2.00 & 3.00 & 3.00 & 3.00 & 2.00 & 3.00 & 2.00 & 2.00 & 2.00 & 2.00 & 2.00 \\
\hline PTI & 2.00 & 2.50 & 3.50 & 3.50 & 3.50 & 2.00 & 3.50 & 2.00 & 3.50 & 4.00 & 2.50 & 3.50 \\
\hline IVI & 2.00 & 2.00 & - & 4.00 & 4.00 & 3.00 & 3.00 & 2.00 & 3.00 & 4.00 & 4.00 & 4.00 \\
\hline Rideshar & 4.00 & 4.00 & 4.00 & 4.00 & 4.00 & 2.00 & 4.00 & 2.00 & 4.00 & 4.00 & 2.00 & 4.00 \\
\hline Software & 4.00 & 4.00 & 4.00 & 4.00 & 4.00 & 2.00 & 4.00 & 2.00 & 2.00 & 2.00 & 2.00 & 4.00 \\
\hline ETS & - & - & 3.00 & 3.00 & 4.00 & 4.00 & 3.00 & - & - & 3.00 & 3.00 & 4.00 \\
\hline
\end{tabular}

Averages are taken from responses by suppliers of the transit technologies. The responses are coded: $0=$ strongly disagnee; $1=$ disagree; 2 = neutral; $3=$ agree; $4=$ strongly agree.

AVI = Automatic Vehicle Identification; AVL $=$ Automatic Vehicle Location; $\mathbf{O B C}=$ On Boand Computers; $\mathbf{A P C}=$ Automatic Passenger

Counter; PTI = Pre-Trip Info System; IVI = In Vehicle Info System; Rideshare = Rideshare Matching Software; Software = Transit Operations

Software; ETS = Electronic Ticketing System 
tered the market. Smaller companies are also competing. However, their longterm survival seems uncertain. Based on the data, the larger companies did not focus on specific products or technology areas. Furthermore, certain products, such as collision avoidance systems and electronic ticketing systems, are supplied by the larger companies only.

\section{Conclusions and Recommendations}

This study has developed a structure for APTS technologies that can help in:

- evaluating the operator and traveler impacts of alternative technologies in terms of performance measures;

- selecting the appropriate technologies for field testing and deployment;

- representing knowledge about existing and new transit technologies; and

- modeling or optimizing transit system performance and designing surveys to understand traveler response.

APTS technologies can be defined by their design and application dimensions. The design dimensions are technology features, functions, and performance. The application dimensions are spatial, temporal, and user measures. This structure facilitates planning for APTS technologies as explained below. Technology deployment takes place in an implementation context. The spatial, temporal, and user dimensions of the implementation context are also important determinants of technology impacts. For APTS deployment, the interrelationships between operator and traveler impacts are important. The technology and context dimensions will have direct, indirect, and simultaneous impacts on transit operators and travelers. The impacts can be classified in terms of operator and traveler performance dimensions (and these measures are distinct from technology performance measures) and distribution dimensions. The impacts have spatial, temporal, and user distributions, and they are distinct from technology application dimensions. That is, the impacts may not always occur where the technology is implemented (at the same locations and/or times). Technologies that have direct impacts on traveler decisions (e.g., mode and route) are termed traveler-based, and those with direct impacts on operator decisions (operations and planning) are termed operator-based. 
The structure developed in the study was used to explore the commercial availability of new transit technologies. Survey research showed that technology suppliers had several products that were likely to vary in their direct and indirect impacts on transit operators and travelers. The opinions provided by technology suppliers give some insights into technology impacts. For example, AVL systems were rated highly on improving monitoring and also controlling transit headways while information technologies were rated highly on traveler impacts, i.e., they can increase traveler comfort and satisfaction. Although closely connected, traveler-based technologies are more suitable for sustaining (and possibly increasing) transit riderships, whereas operator-based technologies are likely to improve transit efficiency and service, and reduce costs.

A strategy for implementation of these technologies must consider the broader mix of technology alternatives. Importantly, there is a need to identify APTS technologies that can be mixed to provide the correct balance between operator effectiveness and customer satisfaction. Individually, APTS technologies may be of limited value but, collectively, they may significantly enhance the performance of the transit system and attract travelers. Therefore, the issue of APTS integration is critical.

Before testing, a strategy should be designed to select appropriate technologies. Besides testing technologies individually, the collective testing of a balanced set of operator- and traveler-based technologies is needed. Furthermore, the tests need to be conducted in a set of carefully selected "high impact" implementation contexts. Among traveler-based technologies, it is possible that pretrip transit information systems (including real-time rideshare systems) have relatively greater potential. This is because during the pre-trip stage, travelers have greater flexibility in travel choices and also because such systems are expected to have significant impacts on traveler response to unexpected congestion (Khattak and Le Colletter 1994). Among operator-based technologies, Automatic Vehicle Monitoring systems can improve transit operations, and the information obtained on transit system performance can be used synergistically with a pre-trip information system. The point is that technology selection process should consider 
synergistically testing various operator- and traveler-based technologies. Specifically, the design and application dimensions of various technologies may be systematically varied across implementation contexts and their impacts observed. The joint implementation of two transit technologies may enhance their individual benefits (compared with implementing them separately). Moreover, technologies may be mutually customized to enhance their individual benefits. Overall, the interaction effects of technologies may be significant and need to be explored.

There are several projects aimed at testing advanced technologies throughout the United States. Following are some guidelines for systematically and consistently evaluating APTS technologies in "high impact" implementation contexts:

1. Develop selection criteria for technologies and impact dimensions, e.g., - policy relevance of impact measures and specific APTS technology solutions;

- design dimensions (features, functions and performance) and application dimensions (space, time and user) of APTS technologies;

- appropriate mix of operator- and traveler-based technologies;

- potential interaction of various technologies if jointly deployed;

- extent and nature of desired impacts on transit operators and travelers;

- compatibility of the new technology with the implementation context and existing transit system; and

- funding and financing opportunities for technology testing.

2. Select candidate technologies for field testing.

3. Design the experiment and develop methodology for evaluation:

- conduct pre-experiment analysis;

- conduct traveler behavior and operator performance surveys; and

- collect and analyze transit system performance and traveler data before and after testing.

4. Test the technology in different contexts (if possible).

5. Systematically evaluate impacts in terms of performance measures and distribution across space, time, and users. 
6. Synthesize outcomes of field experiments:

- direct, indirect, and simultaneous impacts of advanced transit technologies on transit operators and travelers; and

- improvements in design and application dimensions of specific technologies and synergies among technologies.

To synthesize knowledge from APTS field tests, Case-Based Reasoning (CBR) can be used (Khattak and Kanafani 1995). In CBR, the decision to deploy a set of APTS technologies can be made by examining similar historical cases. Rough estimates of traveler and operator impacts for proposed APTS technology "bundles" can be based on previous experiences with similar bundles. All historical cases will be structured according to (a) the design and application dimensions of APTS technologies, (b) the performance and distribution dimensions of impacts, and (c) spatial, temporal and user dimensions of the implementation context. In addition, cases will contain information about lessons learned, such as inferences regarding their success or failure, prescriptions, and case quality. Information about impacts in historical cases can be retrieved by matching the desired and historical bundle of APTS technologies. Increasing similarity of current and historical situations will require (a) matching the desired APTS technology bundle on the design and application dimensions of historical APTS technologies and (b) matching dimensions of the current implementation context with historical implementation contexts. Based on the impacts in historical cases, decisionmakers can infer the extent of impacts in their current situation.

Of course, the selection of new transit technologies and their testing/evaluation in real-life situations will be iterative and semi- or un-structured. The key point is that we need a systematic strategy to determine the value of new transit technologies and to avoid a muddled and opportunistic transit technology testing process that can result in sloppy research, inconsistent conclusions, and inappropriate APTS deployment.

\section{Acknowledgments}

Funding for this study was provided by the California Department of Transportation through the California PATH Program. Professor Randolph Hall has contributed sig- 
nificantly to the development of this study. Mr. Robert Ratcliff's support at Caltrans is appreciated. Ms. Neeloufar Hemassi's help in the analysis and editing was crucial. Finally, we have received valuable ideas from Professor Adib Kanafani.

\section{References}

Ball W. 1994. Commuting Alternatives in the United States: Recent Trends and a Look to the Future, Office of University Research and Education, Research and Special Programs Administration, DOT-T-95-11, Washington, D.C.

Casey R., and J. Collura. 1993. Advanced Public Transportation Systems: Evaluation Guidelines, Federal Transit Administration, US Department of Transportation, FTAMA-26-0007-94-2, Washington, D.C.

Pisarski, A. 1992. Travel Behavior Issues in the 90's, Prepared for Office of Highway Information and Management, HPM-40, USDOT, FHWA, Washington, D.C.

Khattak, A., H. Noeimi, H. Al-Deek, and R. Hall. 1993. Advanced Public Transportation Systems: A Taxonomy and Commercial Availability, Partners in Advanced Transit and Highways (PATH) Research Report UCB-ITS-PRR-93-9, Institute of Transportation Studies, University of California at Berkeley, California.

Khattak, A., and E. Le Colletter. 1994. Stated and Reported Diversion to Public Transportation under Incident Conditions: Implications on the Benefits of Multimodal ATIS, Partners for Advanced Transit and Highways (PATH) Research Report UCBITS-PRR-94-14, Institute of Transportation Studies, University of California at Berkeley, California, 1993, Presented at the 4th Annual Meeting of IVHS America, Atlanta, Georgia.

Khattak, A., and A. Kanafani. 1995. PLANiTS: The Case-Based Reasoner as a Planning Tool for Intelligent Transportation Systems, Partners for Advanced Transit and Highways (PATH) Research Report UCB-ITS-PRR-95-23, Institute of Transportation Studies, University of California at Berkeley, California (forthcoming in Transportation Research C).

\section{About the Authors}

ASAD J. Khattak is Professor in the Department of City and Regional Planning at the University of North Carolina at Chapel Hill. Hisham NoEIMI is a consultant with JRH Transportation Engineering. Haitham M. Al-Deek is Professor with the Department of Civil Engineering at the University of Central Florida in Orlando. 